\title{
Advanced stage ovarian cancer patients with neoadjuvant chemotherapy suffered worse recurrence free survival
}

\section{Cailiang Wu}

Department of Obstetrics and Gynecology, Shanghai General Hospital, Shanghai Jiao Tong University School of Medicine

\section{Xuexin Zhou}

Department of Obstetrics and Gynecology, Shanghai General Hospital, Shanghai Jiao Tong University School of Medicine

\section{Yiwen Feng}

Department of Obstetrics and Gynecology, Shanghai General Hospital, Shanghai Jiao Tong University School of Medicine

Yi Miao

Department of Obstetrics and Gynecology, Shanghai General Hospital, Shanghai Jiao Tong University School of Medicine

\section{Ye Yang}

Department of Obstetrics and Gynecology, Shanghai General Hospital, Shanghai Jiao Tong University School of Medicine

\section{Long Zhou}

Department of Obstetrics and Gynecology, Shanghai General Hospital, Shanghai Jiao Tong University School of Medicine

\section{Xing Li}

Department of Obstetrics and Gynecology, Shanghai General Hospital, Shanghai Jiao Tong University School of Medicine

\section{Xiaoning Ji}

Department of Obstetrics and Gynecology, Shanghai General Hospital, Shanghai Jiao Tong University School of Medicine

\section{ZhenYu Sang}

Department of Obstetrics and Gynecology, Shanghai General Hospital, Shanghai Jiao Tong University School of Medicine

\section{Wei Xu}

Department of Obstetrics and Gynecology, Shanghai General Hospital, Shanghai Jiao Tong University School of Medicine

\section{Yinyan He}


Department of Obstetrics and Gynecology, Shanghai General Hospital, Shanghai Jiao Tong University School of Medicine

\section{Yaping Zhu}

Department of Obstetrics and Gynecology, Shanghai General Hospital, Shanghai Jiao Tong University School of Medicine

\section{Wei Bao ( $\nabla$ forever_chipper@hotmail.com )}

Shanghai General Hospital

\section{Research}

Keywords: Ovarian cancer, Adjuvant chemotherapy, Recurrence

Posted Date: January 9th, 2020

DOI: https://doi.org/10.21203/rs.2.20542/v1

License: (c) (1) This work is licensed under a Creative Commons Attribution 4.0 International License. Read Full License 


\section{Abstract}

\section{Background}

Neoadjuvant chemotherapy (NACT) has been applied for the treatment of patients with advanced-stage epithelial ovarian cancer (EOC), fallopian tube cancer, and primary peritoneal cancer, as these patients have a low likelihood of achieving optimal debulking and are thus poor surgical candidates. Herein, we explore the effects of NACT and compare the surgical outcomes and recurrence data in patients who receive interval debulking surgery followed NACT(NACT-IDS) or primary debulking surgery(PDS).

Methods

A retrospective, single-center, observational study was conducted. Patients with advanced-stage EOC, fallopian tube cancer and primary peritoneal cancer who were treated with NACT or primary debulking surgery were enrolled. The effects of NACT as well as the surgical outcomes and recurrence data were compared between the NACT-IDS and PDS groups.

Results

The albumin level was elevated $(42.61 \pm 3.46 \mathrm{~g} / \mathrm{L}$ vs. $37.47 \pm 5.42 \mathrm{~g} / \mathrm{L}, \mathrm{P}=0.001)$ and the levels of CA12-5 and HE4 significantly decreased $(P=0.002,0.003)$ in patients after neoadjuvant courses. The operation time, amount of blood loss during surgery, rate of bowel resection, time to chemotherapy, and platinumfree interval were comparable between the two groups (P凶0.05). Recurrence-free survival was worse in the NACT-IDS group than in the PDS group (HR=2.406, 95\% CI[1.024, 5.657]).

\section{Conclusion}

NACT improved the condition of advanced-stage patients, but a poor recurrence free survival rate was observed; thus, NACT should not be applied in non-selected patients.

\section{Background}

Epithelial ovarian cancer (EOC) is the fifth most common type of cancer and the leading cause of death due to cancer in women; there were 295,414 newly diagnosed cases and 184,799 deaths in 2018 worldwide ${ }^{1}$, and there were 22,530 cases and 13,980 deaths in 2019 in the United States ${ }^{2}$. The standard management of ovarian cancer is a combination of cytoreductive surgery (primary debulking surgery) followed by platinum-based chemotherapy. The purpose of surgery is ideally to remove the tumor, with less than or equal to $1 \mathrm{~cm}$ of residual tumor, which is associated with a survival benefit ${ }^{3}$. However, more than $75 \%$ of OC patients diagnosed with advanced-stage disease have accompanying ascites and hypoalbuminemia, which are predictive of severe postoperative complications ${ }^{4,5}$, and complications that occur after surgery delay adjuvant chemotherapy ${ }^{6}$. Therefore, neoadjuvant chemotherapy followed by interval debulking surgery is suggested ${ }^{7}$ for patients with advanced-stage disease who have a low 
likelihood of achieving optimal debulking and thus are poor surgical candidates. In addition, one study found higher quality of life scores in terms of emotional and cognitive function ${ }^{5}$. Importantly, the survival of patients who receive NACT followed by interval debulking surgery has been found to be no worse than the survival of those treated with PDS ${ }^{8,9}$. Recently, the utilization of NACT has been proven to rapidly decrease 30 - and 90 -day mortality after surgery ${ }^{10}$.

In this study, we aimed to explore the effects of NACT and to compare the surgical outcomes and longterm recurrence information between an NACT-IDS and a PDS group.

\section{Methods}

\section{Patient selection}

Patients with epithelial ovarian cancer, fallopian tube cancer or primary peritoneal cancer diagnosed by a pathologist and treated with NACT followed by interval debulking surgery (NACT-IDS) from January 1 , 2013 , to October 31, 2018, were considered potential participants. We also selected patients treated with primary debulking surgery (PDS) followed by platinum-based adjuvant chemotherapy during the same period for inclusion in the control group. The exclusion criteria were as follows: patients diagnosed with other malignant tumors and patients who did not undergo interval debulking surgery after NACT, or loss of follow-up. Informed consent was obtained.

We collected general information, including age, histology type, FIGO stage, and hemoglobin, albumin, alanine aminotransferase, aspartate aminotransferase, CA12-5, and HE4 levels before and after neoadjuvant therapy/surgery. We also collected information on the duration of surgery and the volume of bleeding during surgery as well as information regarding recurrence, platinum-free interval, and recurrence-free survival.

\section{Follow-up}

The follow-up period ended on December 31, 2019, and all included participants underwent regular tests for serum CA12-5 levels and abdominal/pelvic computerized tomography(CT)/ magnetic resonance imaging(MRI). Recurrence was defined as elevated serum CA12-5 levels ( $₫ 35 \mathrm{U} / \mathrm{ml}$ ) as assessed by $\mathrm{CT} / \mathrm{MRI}$ findings. The recurrence free survival was calculated from date of surgery to date of recurrence, and the platinum-free interval was calculated from the last chemotherapy cycle to the time of recurrence.

\section{Statistical analysis}

SPSS 19.0 was applied for the statistical analysis, and Pख0.05 was considered statistically significant. We used means \pm standard deviations (SD) or medians (interquartile ranges) to express quantitative variables, and qualitative variables were expressed as absolute numbers (percentages). Student's unpaired $t$ test for variables with a normal distribution and the Mann-Whitney $U$ test for variables with a 
nonnormal distribution were used for the comparison of quantitative variables between the groups, and the Chi-square test was used for qualitative variables. Kaplan-Meier survival analysis was used to analyze recurrence free survival with GraphPad Prism 8.0.

\section{Results}

\section{Clinical characteristics of the participators}

Twenty-five patients received NACT followed by IDS, and 22 patients treated with PDS as the initial treatment were included. The baseline characteristics of the included patients are listed in Table 1. The age, FIGO stage, histology type, adjuvant chemotherapy cycle and rate of bowel resection were comparable between the NACT and PDS groups, while the diameters of the mass were larger in the PDS group than in the NACT group $(P=0.000)$.

Table 1

Characteristics of the participated patients

\begin{tabular}{|c|c|c|c|}
\hline & NACT $(n=25)$ & PDS $(n=22)$ & $\mathbf{P}$ \\
\hline Age $(y)$ & $58.96 \pm 9.87$ & $56.64 \pm 10.64$ & 0.442 \\
\hline FIGO stage $(n, \%)$ & & & 0.578 \\
\hline 『c & 1 & 3 & \\
\hline IIIC & 22 & 16 & \\
\hline IV & 2 & 3 & \\
\hline Histology type $(\mathrm{n}, \%)$ & & & 0.105 \\
\hline Serous & 22 & 14 & \\
\hline Other & 3 & 8 & \\
\hline Diameter (mm) & $44.57 \pm 25.74$ & $85.94 \pm 37.35$ & 0.000 \\
\hline Neoadjuvant cycle & $3(2,3)$ & - & - \\
\hline Adjuvant cycle & $5.60 \pm 2.33$ & $5.43 \pm 1.86$ & 0.787 \\
\hline Bowel resection $(n, \%)$ & $6,24 \%$ & $4,18.18 \%$ & 0.627 \\
\hline Length of follow-up, (Mean $\pm S D$ ) & $984.08 \pm 543.62$ & $758.41 \pm 202.44$ & 0.063 \\
\hline
\end{tabular}

\section{The effect of NACT}

In the NACT group, serum markers were selected to evaluate the effect of NACT before and after the course of chemotherapy. Additionally, we found that the albumin level was elevated after chemotherapy 
$(P=0.001)$, while the CA12-5 and HE4 levels were obviously decreased posttreatment $(P=0.002,0.003)$. In addition, the AST and ALT levels were comparable before and after the invention $(P=0.194,0.074$; Table 2).

Table 2

The serum biomarkers tested pre- and post-NACT

\begin{tabular}{|llll|}
\hline & PreNACT & Pos-NACT & P \\
\hline Albumin, mean, SD $(\mathrm{g} / \mathrm{L})$ & $37.47 \pm 5.42$ & $42.61 \pm 3.46$ & 0.001 \\
\hline Hemoglobin, mean, SD $(\mathrm{g} / \mathrm{L})$ & $120.36 \pm 15.91$ & $105.61 \pm 13.36$ & 0.000 \\
\hline CA 12 - 5, mean, SD $(\mathrm{U} / \mathrm{ml})$ & $1610.52 \pm 1677.53$ & $215.69 \pm 316.39$ & 0.002 \\
\hline HE4, median range $(\mathrm{U} / \mathrm{ml})$ & $471.70(77.12,1527.24)$ & $112.40(38.81,1272.00)$ & 0.003 \\
\hline ASL, mean, SD (U/L) & $26.11 \pm 13.78$ & $30.58 \pm 11.08$ & 0.194 \\
\hline ALT, mean, SD $(\mathrm{U} / \mathrm{L})$ & $19.30 \pm 16.73$ & $26.51 \pm 12.35$ & 0.074 \\
\hline
\end{tabular}

\section{The surgical outcomes and recurrence data of NACT-IDS and PDS}

The preoperative hemoglobin and HE4 levels were lower in the NACT-IDS group than in the PDS group (P $=0.001, P=0.031)$, and the AST and ALT levels were higher in the PDS group than in the NACT-IDS group $(P=0.005, P=0.040)$; the operation time, blood loss during surgery, rate of bowel resection, and time from surgery to initial chemotherapy were comparable ( $\mathrm{P} \otimes 0.05)$. After the operation, the levels of hemoglobin and CA12-5 in the NAST-IDS group were lower than those in the PDS group $(P=0.022, P=0.045)$. During the follow-up period, there were 14 patients with recurrence in the NACT group and 9 in the PDS group (P $=0.302$ ). Among the patients who had recurrence, the platinum-free interval was $183.86 \pm 89.28$ days in the NACT-IDS group and $288.75 \pm 188.56$ days in the PDS group $(P=0.173)$. Additionally, there were $6 / 14$ (42.86\%) patients in the NACT-IDS group whose platinum-free interval was $<6$ months and $2 / 9(22.22 \%)$ patients in the PDS group $(P=0.333$ ) (Table 3$)$. Kaplan-Meier survival analysis for recurrence free survival demonstrated that patients who received NACT followed by IDS had a poor recurrence free survival $(P=$ 0.0441). (Fig. 1) 
Table 3

The surgical outcomes and recurrence data of NACT-IDS and PDS

\begin{tabular}{|llll|}
\hline & NACT $(\mathbf{n}=\mathbf{2 5})$ & PDS $(\mathbf{n}=\mathbf{2 2})$ & P \\
\hline Operation time $(\mathrm{min})$ & $216.08 \pm 61.23$ & $240.82 \pm 60.55$ & 0.176 \\
\hline Blood loss $(\mathrm{ml})$ & $300.00(200.00,500.00)$ & $300.00(200.00,600.00)$ & 0.286 \\
\hline Bowel resection $(\mathrm{n})$ & $6(24.00 \%)$ & $4(18.18 \%)$ & 0.897 \\
\hline Time to chemotherapy (day) & $11.00(9.00,18.50)$ & $11.00(10.00,14.75)$ & 0.792 \\
\hline Recurrence, $\mathrm{n}, \%$ & $14(56.00 \%)$ & $8(36.36 \%)$ & 0.178 \\
\hline Platinum-free interval (<6 months), $\mathrm{n}, \%$ & $6(24.00 \%)$ & $2(9.09 \%)$ & 0.333 \\
\hline Platinum-free interval, day, mean \pm SD & $183.86 \pm 89.28$ & $288.75 \pm 188.56$ & 0.173 \\
\hline
\end{tabular}

\section{Discussion}

Ovarian cancer is the leading cause of death from gynecological malignancy worldwide, as most patients are diagnosed at advanced stages due to a lack of sensitive screening tests and the lack of specific symptoms at early stages. The standard of treatment is surgery followed by platinum-based chemotherapy ${ }^{11}$, and optimal debulking is the goal, as residual tumors decrease progression-free survival and overall survival ${ }^{12}$. To achieve the goal of optimal debulking, multivisceral surgeries such as bowel section and splenectomy are performed ${ }^{13,14}$.

Invasive procedures are associated with high risks of morbidity and mortality; in addition, poor outcomes, such as hypoalbuminemia, are predictive of severe postoperative complications ${ }^{4}$, and complications delay the start of subsequent chemotherapy, which reduces progression-free survival ${ }^{6}$. Therefore, neoadjuvant chemotherapy offers an alternative treatment for patients with advanced ovarian cancer and makes surgery highly feasible ${ }^{15}$. In our study, we found that the level of albumin was elevated after the course of NACT ( $37.47 \pm 5.42$ vs $42.61 \pm 3.46$, P $₫ 0.05$ ). Cancer patients have low preoperative albumin levels, especially those with advanced-stage disease and patients with ascites ${ }^{16}$; the mechanisms for this low level involve a decrease in amino acid intake and albumin synthesis ${ }^{17}$ as well as an increase in albumin leakage to the extravascular space in patients with ascites. There were 21 (80.77\%) patients with massive ascites upon diagnosis, and the largest mean diameter of the ascites assessed by ultrasound was $104.05 \pm 31.75 \mathrm{~mm}$. After the course of NACT, ascites was not detected in 3 patients, and the largest mean diameter of the remaining cases of ascites was $65.00 \pm 35.32 \mathrm{~mm}(\mathrm{t}=3.636, \mathrm{P}=0.001)$. Furthermore, decreases in CA12-5 and HE4 levels were found (P凶0.05) in the NACT group, and side effects of chemotherapy (lowered hemoglobin levels) also occurred. Previous studies have observed that patients who receive interval debulking surgery after NACT have better surgical outcomes than patients who receive PDS, including less severe surgical complexity, fewer upper abdominal procedures, a shorter 
surgical duration, a lower amount of perioperative blood loss, a shorter hospital stay ${ }^{5,8,18}$, and reduced surgical morbidity and mortality 7,8 . In our study, there was no difference in the surgical outcomes between the NACT and PDS groups, as NACT may induce fibrosis, which may influence surgical procedures.

The presence of residual disease after surgery largely impact the prognosis of EOC ${ }^{19}$; one study showed that the 7 -year overall survival rate decreased from $73.6 \%$ in patients without residual tumors to $21 \%$ in patients with any type of residual disease ${ }^{20}$. Therefore, the goal of debulking surgery has been changed from the presence of a residual tumor less than $1 \mathrm{~cm}$ in diameter to the presence of no visible tumor ${ }^{21}$; however, advanced-stage disease is associated with a low rate of complete tumor resection ${ }^{22}$. A previous study demonstrated that patients who received IDS after NACT were likely to achieve optimal cytoreduction ${ }^{20,23}$ but that they did not have superior survival ${ }^{24}$, and there was a decreasing trend in median survival with an increase in the number of NACT cycles ${ }^{24,25}$. These studies suggest that NACT impacts the evaluation of tumor spread and leads to incomplete tumor resection in potentially resectable areas.

In addition, patients treated with NACT-IDS suffer from recurrence more often than patients treated with PDS $^{26}$. In our study, during the follow-up period, 14 (56.00\%) patients experienced recurrence in the NACT group, and $8(36.36 \%)$ had recurrence in the PDS group; the difference was not significant (Pख0.05). However, recurrence-free survival was poorer in the NACT-IDS group than in the PDS group (HR 2.406, 95\% $\mathrm{Cl}[1.024,5.657])$. Rauh-Hain and colleagues found that $88.8 \%$ of cases of recurrence in the NACT-IDS group were platinum resistant compared to $55.3 \%$ of cases in the PDS group $(P=0.001)^{27}$. The same outcome was also found by Gao, who showed that $40.3 \%$ of patients in the NACT-IDS group and $23.1 \%$ in the PDS group experienced platinum-resistant recurrence and ${ }^{28}$; similarly, NACT induced chemotherapy resistance in cancer stem cell colonies ${ }^{29}$. A limited number of studies have found that the TP53 gene mutation is the most common (70.3\%) mutation after courses of NACT compared with before courses of $\mathrm{NACT}^{30}$; additionally, in platinum-resistant patients (relapsed within 6 months), the TP53 K351N mutation was significantly more severe in the NACT-IDS group than in the PDS group ( $57.14 \%$ vs $0 \%, P \llbracket 0.01)$. Even so, in our study, we found that there was no significant difference in the platinum-free interval (164.93 \pm 94.65 vs. $288.75 \pm 188.56, \mathrm{P}=0.1115)$ or in the $<6$-month $\mathrm{PFI}(6 / 25$ vs. $2 / 22, \mathrm{P}=0.333)$.

There were some limitations in this study. First, this was a retrospective analysis in a single institution with a small sample size; thus, much stronger evidence is needed to confirm the results. Additionally, due to limited information, we did not compare overall survival between the two groups.

\section{Conclusion}

NACT improves the performance of patients, but patients suffered more recurrence during the follow-up period. NACT should not be applied in all advanced ovarian cancer patients without selected. 


\section{Abbreviations}

NACT: neoadjuvant chemotherapy; IDS: interval debulking surgery; PDS: primary debulking surgery; CT: computerized tomography; MRI: magnetic resonance imaging; SD: standard deviations; Cl: confidence interval

\section{Declarations}

\section{Acknowledgements}

None

\section{Authors' contributions}

Cailiang Wu and Xuexin Zhou equally contribution to this manuscript, acquisition of data, analysis, and drafting the article. Yaping Zhu and Wei Bao contributed to the design of the study. Wei Xu, Yinyan He and Zhenyu Sang: interpretation of data. Cailiang Wu, Xuexin Zhou, Yi Miao, Long Zhou and Yiwen Feng: contribution to the concept of the study, coordinated the study. Cailiang Wu, Xuexin Zhou, Xing Li and Xiaoning Ji: contributed to the study execution and analysis.

\section{Authors' details}

1 Department of gynaecology and obstetrics, Shanghai General Hospital, Shanghai 200080, China

\section{Availability of data and materials}

The data and materials used in the present study are available from the corresponding authors.( Also showed in Supplementary File)

\section{Consent for publication}

All of the authors read the final manuscript and consent for publication

\section{Competing interests}

None declared

\section{Funding}

The present study was sponsored by the National Natural Science Foundation of China (nos. 81972425), the Shanghai PuJiang Program (no. 17PJD032) and the Project Young Elite of the Shanghai Health System (no. 2017YQ063).

\section{Ethics approval and consent to participate}


Our study approved by the Ethics committee of Shanghai General Hospital, and all the informed consent of included patients obtained.

\section{References}

1. Bray F, Ferlay J, Soerjomataram I, Siegel RL, Torre LA, Jemal A. Global cancer statistics 2018: GLOBOCAN estimates of incidence and mortality worldwide for 36 cancers in 185 countries. CA Cancer J Clin. 2018;6:394-424.

2. Siegel RL, Miller KD, Jemal A. Cancer statistics, 2019. CA Cancer J Clin. 2019;1:7-34.

3. du Bois A, Reuss A, Pujade-Lauraine E, Harter P, Ray-Coquard I, Pfisterer J. Role of surgical outcome as prognostic factor in advanced epithelial ovarian cancer: a combined exploratory analysis of 3 prospectively randomized phase 3 multicenter trials: by the Arbeitsgemeinschaft Gynaekologische Onkologie Studiengruppe Ovarialkarzinom (AGO-OVAR) and the Groupe d'Investigateurs Nationaux Pour les Etudes des Cancers de l'Ovaire (GINECO). Cancer. 2009;6:1234-1244.

4. Ataseven B, du Bois A, Reinthaller A, Traut A, Heitz F, Aust S, Prader S, et al. Pre-operative serum albumin is associated with post-operative complication rate and overall survival in patients with epithelial ovarian cancer undergoing cytoreductive surgery. Gynecol Oncol. 2015;3:560-565.

5. Fagotti A, Ferrandina G, Vizzielli G, Fanfani F, Gallotta V, Chiantera V, Costantini B, et al. Phase III randomised clinical trial comparing primary surgery versus neoadjuvant chemotherapy in advanced epithelial ovarian cancer with high tumour load (SCORPION trial): Final analysis of peri-operative outcome. Eur J Cancer. 2016:22-33.

6. Hofstetter G, Concin N, Braicu I, Chekerov R, Sehouli J, Cadron I, Van Gorp T, et al. The time interval from surgery to start of chemotherapy significantly impacts prognosis in patients with advanced serous ovarian carcinoma - analysis of patient data in the prospective OVCAD study. Gynecol Oncol. 2013;1:15-20.

7. Wright AA, Bohlke K, Armstrong DK, Bookman MA, Cliby WA, Coleman RL, Dizon DS, et al. Neoadjuvant Chemotherapy for Newly Diagnosed, Advanced Ovarian Cancer: Society of Gynecologic Oncology and American Society of Clinical Oncology Clinical Practice Guideline. J Clin Oncol. 2016;28:3460-3473.

8. Kehoe S, Hook J, Nankivell M, Jayson GC, Kitchener H, Lopes T, Luesley D, et al. Primary chemotherapy versus primary surgery for newly diagnosed advanced ovarian cancer (CHORUS): an open-label, randomised, controlled, non-inferiority trial. Lancet. 2015;9990:249-257.

9. Onda T, Satoh T, Saito T, Kasamatsu T, Nakanishi T, Nakamura K, Wakabayashi M, et al. Comparison of treatment invasiveness between upfront debulking surgery versus interval debulking surgery following neoadjuvant chemotherapy for stage III/IV ovarian, tubal, and peritoneal cancers in a phase III randomised trial: Japan Clinical Oncology Group Study JCOG0602. Eur J Cancer. 2016:2231.

10. Horner W, Peng K, Pleasant V, Brackmann M, Ebott J, Gutfreund R, McLean K, et al. Trends in surgical complexity and treatment modalities utilized in the management of ovarian cancer in an era of 
neoadjuvant chemotherapy. Gynecol Oncol. 2019;2:283-289.

11. Morotti M, Becker CM, Menada MV, Ferrero S. Targeting tyrosine-kinases in ovarian cancer. Expert Opin Investig Drugs. 2013;10:1265-1279.

12. Rose PG, Nerenstone S, Brady MF, Clarke-Pearson D, Olt G, Rubin SC, Moore DH, et al. Secondary surgical cytoreduction for advanced ovarian carcinoma. N Engl J Med. 2004;24:2489-2497.

13. Tozzi R, Hardern K, Gubbala K, Garruto Campanile R, Soleymani Majd H. En-bloc resection of the pelvis (EnBRP) in patients with stage IIIC-IV ovarian cancer: A 10 steps standardised technique. Surgical and survival outcomes of primary vs. interval surgery. Gynecol Oncol. 2017;3:564-570.

14. Soleymani Majd H, Ferrari F, Manek S, Gubbala K, Campanile RG, Hardern K, Tozzi R. Diaphragmatic peritonectomy vs. full thickness resection with pleurectomy during Visceral-Peritoneal Debulking (VPD) in 100 consecutive patients with stage IIIC-IV ovarian cancer: A surgical-histological analysis. Gynecol Oncol. 2016;3:430-435.

15. Worley MJ, Jr., Guseh SH, Rauh-Hain JA, Williams KA, Muto MG, Feltmate CM, Berkowitz RS, et al. Does neoadjuvant chemotherapy decrease the risk of hospital readmission following debulking surgery? Gynecol Oncol. 2013;1:69-73.

16. Ayhan A, Günakan E, Alyazıcı İ, Haberal N, Altundağ Ö, Dursun P. The preoperative albumin level is an independent prognostic factor for optimally debulked epithelial ovarian cancer. Arch Gynecol Obstet. 2017;5:989-995.

17. Ballmer PE, Ochsenbein AF, Schütz-Hofmann S. Transcapillary escape rate of albumin positively correlates with plasma albumin concentration in acute but not in chronic inflammatory disease. Metabolism. 1994;6:697-705.

18. Zhang H, Wang Y, Jiang Z-M, Kondrup J, Fang H, Andrews M, Nolan MT, et al. Impact of nutrition support on clinical outcome and cost-effectiveness analysis in patients at nutritional risk: A prospective cohort study with propensity score matching. Nutrition. 2017:53-59.

19. Winter WE, 3rd, Maxwell GL, Tian C, Carlson JW, Ozols RF, Rose PG, Markman M, et al. Prognostic factors for stage III epithelial ovarian cancer: a Gynecologic Oncology Group Study. J Clin Oncol. 2007; $24: 3621-3627$.

20. Rosen B, Laframboise S, Ferguson S, Dodge J, Bernardini M, Murphy J, Segev Y, et al. The impacts of neoadjuvant chemotherapy and of debulking surgery on survival from advanced ovarian cancer. Gynecol Oncol. 2014;3:462-467.

21. Chang S-J, Bristow RE. Evolution of surgical treatment paradigms for advanced-stage ovarian cancer: redefining 'optimal' residual disease. Gynecol Oncol. 2012;2:483-492.

22. Polterauer S, Vergote I, Concin N, Braicu I, Chekerov R, Mahner S, Woelber L, et al. Prognostic value of residual tumor size in patients with epithelial ovarian cancer FIGO stages IIA-IV: analysis of the OVCAD data. Int J Gynecol Cancer. 2012;3:380-385.

23. Fagö-Olsen CL, Ottesen B, Kehlet H, Antonsen SL, Christensen IJ, Markauskas A, Mosgaard BJ, et al. Does neoadjuvant chemotherapy impair long-term survival for ovarian cancer patients? A nationwide Danish study. Gynecol Oncol. 2014;2:292-298. 
24. Ren Y, Shi T, Jiang R, Yin S, Wang P, Zang R. Multiple Cycles of Neoadjuvant Chemotherapy Associated With Poor Survival in Bulky Stage IIIC and IV Ovarian Cancer. Int J Gynecol Cancer. 2015;8:1398-1404.

25. Bristow RE, Chi DS. Platinum-based neoadjuvant chemotherapy and interval surgical cytoreduction for advanced ovarian cancer: a meta-analysis. Gynecol Oncol. 2006;3:1070-1076.

26. Himoto Y, Cybulska P, Shitano F, Sala E, Zheng J, Capanu M, Nougaret S, et al. Does the method of primary treatment affect the pattern of first recurrence in high-grade serous ovarian cancer? Gynecol Oncol. 2019;2:192-200.

27. Rauh-Hain JA, Nitschmann CC, Worley MJ, Jr., Bradford LS, Berkowitz RS, Schorge JO, Campos SM, et al. Platinum resistance after neoadjuvant chemotherapy compared to primary surgery in patients with advanced epithelial ovarian carcinoma. Gynecol Oncol. 2013;1:63-68.

28. Gao Y, Li Y, Zhang C, Han J, Liang H, Zhang K, Guo H. Evaluating the benefits of neoadjuvant chemotherapy for advanced epithelial ovarian cancer: a retrospective study. J Ovarian Res. 2019;1:85-85.

29. Lim MC, Song YJ, Seo S-S, Yoo C-W, Kang S, Park S-Y. Residual cancer stem cells after interval cytoreductive surgery following neoadjuvant chemotherapy could result in poor treatment outcomes for ovarian cancer. Onkologie. 2010;6:324-330.

30. Lee YJ, Kim D, Shim JE, Bae S-J, Jung Y-J, Kim S, Lee H, et al. Genomic profiling of the residual disease of advanced high-grade serous ovarian cancer after neoadjuvant chemotherapy. Int $\mathrm{J}$ Cancer. 2019:10.1002/ijc.32729.

\section{Figures}




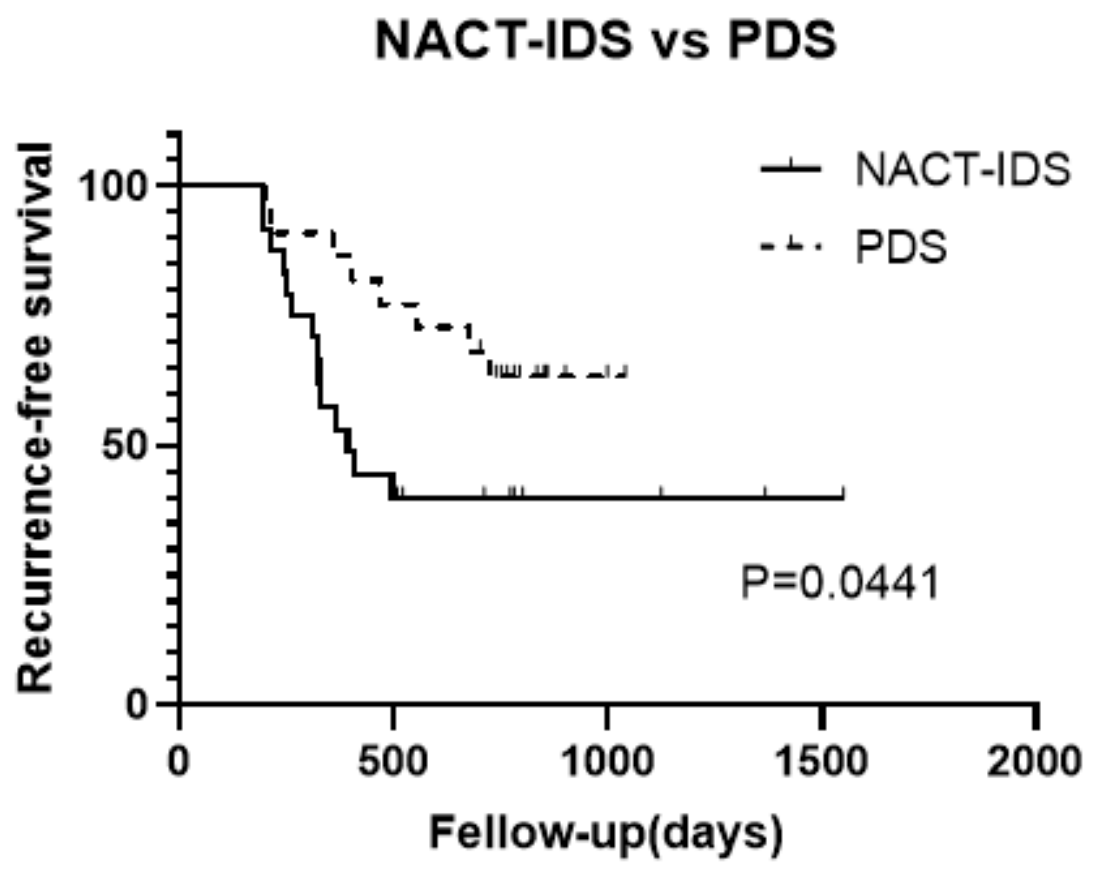

Figure 1

The Kaplan-Meier curve for RFS

\section{Supplementary Files}

This is a list of supplementary files associated with this preprint. Click to download.

- nact.xlsx 